\title{
Competing effect of wind braking and interior coupling in the rotational evolution of solar-like stars
}

\author{
F. Spada ${ }^{1}$ and A. C. Lanzafame $e^{2,3}$ \\ ${ }^{1}$ Max-Planck-Institut für Sonnensystemforschung, Justus-von-Liebig-Weg 3, 37077 Göttigen, Germany
e-mail: spada@mps . mpg.de
2 Università di Catania, Dipartimento di Fisica e Astronomia, Sezione Astrofisica, Via S. Sofia 78, 95123 Catania, Italy
3 INAF-Osservatorio Astrofisico di Catania, Via S. Sofia 78, 95123 Catania, Italy
}

Received 26 July 2019 / Accepted 9 March 2020

\begin{abstract}
Solar-like stars $\left(M \lesssim 1.3 M_{\odot}\right)$ lose angular momentum through their magnetized winds. The resulting evolution of the surface rotation period, which can be directly measured photometrically, has the potential to be an accurate indicator of stellar age, and is constrained by observations of rotation periods of coeval stars, such as members of Galactic open clusters. A prominent observational feature of the mass-rotation period diagrams of open clusters is a sequence of relatively slower rotators. The formation and persistence of this slow-rotator sequence across several billion years imply an approximately coherent spin-down of the stars that belong to it. In particular, the sequence is observed to evolve coherently toward longer periods in progressively older clusters. Recent observations of the $\approx 700$ Myr Praesepe and the 1 Gyr NGC 6811 clusters, however, are not fully consistent with this general pattern. While the stars of $1 M_{\odot}$ on the slow-rotator sequence of the older NGC 6811 have longer periods than their counterparts in the younger Praesepe, as expected, the two sequences essentially merge at lower masses $\left(\lesssim 0.8 M_{\odot}\right)$. In other words, it seems that low-mass stars have not been spinning down in the intervening $300 \mathrm{Myr}$. Here we show that this behavior is a manifestation of the variable rotational coupling in solar-like stars. The resurfacing of angular momentum from the interior can temporarily compensate for that lost at the surface due to wind braking. In our model the internal redistribution of angular momentum has a steep mass dependence; as a result, the re-coupling occurs at different ages for stars of different masses. The semi-empirical mass dependence of the rotational coupling timescale included in our model produces an evolution of the slow-rotator sequence in very good agreement with the observations. Our model, in particular, explains the stalled surface spin-down of low-mass stars between Praesepe and NGC 6811, and predicts that the same behavior should be observable at other ages in other mass ranges.
\end{abstract}

Key words. stars: rotation - stars: evolution - stars: late-type - open clusters and associations: individual: Pleiades open clusters and associations: individual: Praesepe - open clusters and associations: individual: NGC 6811

\section{Introduction}

Solar-like stars $\left(M \lesssim 1.3 M_{\odot}\right)$ have outer convection zones where magnetic fields are generated through dynamo action. These stars therefore have magnetically active atmospheres, and the braking torque exerted by their magnetized winds efficiently carries away angular momentum from their surfaces (e.g., Schatzman 1962; Kraft 1967; Weber \& Davis 1967; Kawaler 1988). As a result, in contrast to their more massive counterparts, solar-like stars undergo a significant rotational evolution on the main sequence. For instance, the rotation period of a star of solar mass is observed to be $P_{\text {rot }} \approx 1$ day upon reaching the zero-age main sequence $(40 \mathrm{Myr})$, in contrast with the 26.09 days of the present Sun $(4.57 \mathrm{Gyr})$. The rotation period of solar-like stars, moreover, can be derived accurately from the analysis of their photometric light curves. The availability of a directly measurable quantity undergoing such large variations on the main sequence holds great potential as an accurate age indicator (known as gyrochronology; Barnes 2003; Barnes et al. 2016b).

The most stringent constraints on the rotational evolution of solar-like stars come from $P_{\text {rot }}$ measurements for stars in Galactic open clusters, whose age is independently known from classical methods, such as isochrone fitting (e.g., Demarque \& Larson 1964). The mass-rotation period diagram of a cluster, in analogy to its classical counterpart, the color-magnitude diagram, reveals the (rotational) evolutionary state of a sample of stars of different mass at a fixed age.

While stars in early pre-main sequence clusters (age $\approx 1-$ $10 \mathrm{Myr}$; see, e.g., Moraux et al. 2013) have a broad range of periods with an approximately uniform distribution in mass, in older clusters a clear pattern gradually emerges. A distinct sequence of relatively slower rotators is clearly visible in $\approx 70$ Myr clusters, or older (see Fig. 1 of Barnes 2003).

At its outset, the slow-rotator sequence is well defined only at the high-mass end of the solar-like regime $\left(M \approx 1.3 M_{\odot}\right)$, while at lower masses it coexists with a less structured broad distribution of faster rotators. The fast rotators gradually disappear in clusters of increasing age, as stars of ever lower mass converge to the slow-rotator sequence. By the age of $1 \mathrm{Gyr}$, all stars down to $0.6 M_{\odot}$ have joined the slow-rotator sequence. Although observations suggest that stars of mass $M<0.6 M_{\odot}$ eventually reach the slow-rotator sequence as well, data in sufficiently old clusters and for low masses are still too scarce to draw definitive conclusions (but see Newton et al. 2018, and references therein).

The details of the formation of the slow-rotator sequence are still not well understood, and only semi-empirical models have been proposed so far (Barnes 2010; Brown 2014; 


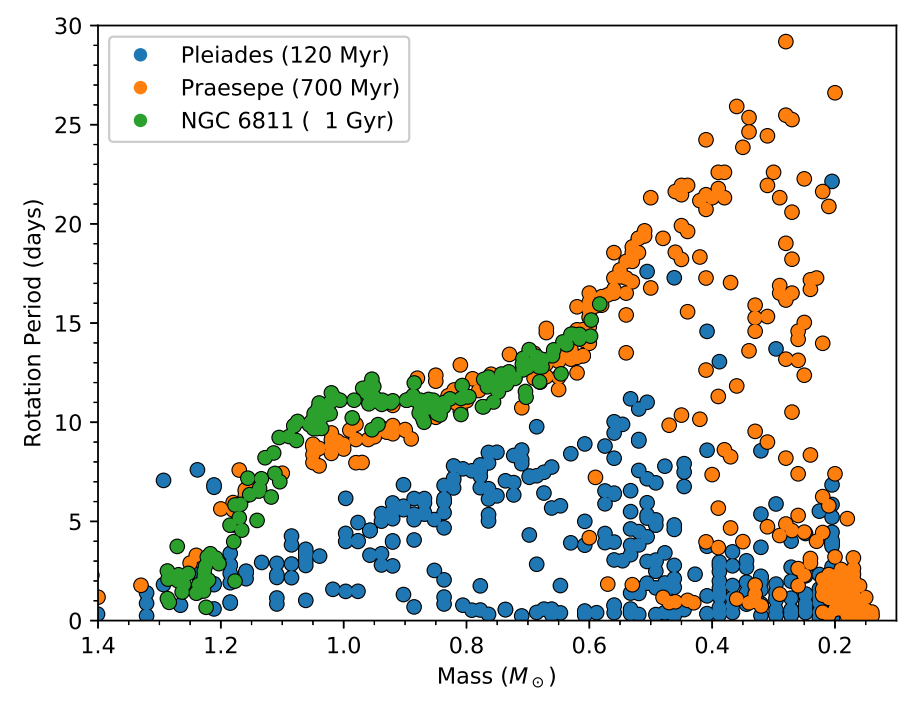

Fig. 1. Emergence and evolution of the slow-rotator sequence in the mass-period diagram of open clusters, from the 120 Myr Pleiades to the 1 Gyr NGC 6811. Data sources: Pleiades: Rebull et al. (2016); Praesepe: Douglas et al. (2019); NGC 6811: Curtis et al. (2019).

Gondoin 2017). The observational evidence summarized above seems to imply that stars settle down on the slow-rotator sequence by means of a quick, one-time transition on a massdependent timescale. Since stars of the same mass do not all converge on the slow-rotator sequence at once, a third parameter (besides mass and age) and/or a stochastic transition from the fast- to slow-rotator regime must be involved.

In addition to the open issue of its mechanism of formation, the persistence of the slow-rotator sequence (clearly observed up to at least $\approx 4 \mathrm{Gyr}$ in M 67: Barnes et al. 2016a) points to an intrinsic regularity in the rotational evolution of stars once they have converged on it. The first attempts to model the evolution of the slow-rotator sequence assumed its mass dependence to be factorizable from the age dependence, and that the age dependence follows the classical spin-down relation proposed by Skumanich (1972), $P_{\text {rot }} \propto \sqrt{t}$ (Barnes 2003, 2007). Although these assumptions were later found to be inadequate (Meibom et al. 2009, 2011; Barnes \& Kim 2010; Lanzafame \& Spada 2015, hereafter LS15), the slow-rotator sequences of clusters of different ages follow a monotonic relation in the mass-rotation period diagram, with older clusters corresponding to slower periods.

The most recent observations of the Praesepe cluster ( $\approx 700$ Myr, Douglas et al. 2019; see also Sect. 2) and NGC 6811 clusters (1 Gyr, Janes et al. 2013), featuring an unprecedented sampling of the low-mass regime of their slow-rotator sequences, seem to contradict this (so far) well-established fact (Curtis et al. 2019; Douglas et al. 2019). While $\approx 1 M_{\odot}$ stars on the slow-rotator sequence of these two clusters display the expected behavior (i.e., the younger Praesepe stars rotating faster than those in the older NGC 6811), the two sequences seem to overlap below $0.8 M_{\odot}$ (see Fig. 1). In other words, low-mass stars in NGC 6811 do not seem to have been spinning down significantly in the intervening $300 \mathrm{Myr}$.

In this paper we model the evolution of the slow-rotator sequence, and in particular we provide a theoretical interpretation of the puzzling new data from Praesepe and NGC 6811. We show that our model, based on our previous work (LS15), can reproduce the apparent "stalled" (or reduced) spin-down observed in NGC 6811, and that this is the result of the redistribution of angular momentum from the stellar interior to the surface, which is temporarily able to offset the effect of the magnetic wind braking. The two key ingredients of the model are the wind braking law and the mass dependence of the rotational coupling timescale originally proposed by LS15. The latter is also in remarkable agreement with the results of Somers \& Pinsonneault (2016), obtained from an independent analysis of a related, but distinct problem (the lithium depletion in solar-like stars).

Our results provide valuable constraints on the mechanisms that transport angular momentum in the interior of solar-like stars, whose physical nature is still uncertain and strongly debated (e.g., Charbonneau \& MacGregor 1993; Ruediger \& Kitchatinov 1996; Charbonnel \& Talon 2005; Spada et al. 2010; Eggenberger et al. 2019). Moreover, we demonstrate that the assumption of a solid-body rotation profile in stellar interiors is inadequate to obtain accurate gyrochronology relations.

This paper is organized as follows: we describe the observational data used to constrain our models in Sect. 2; we outline the physics of our rotational evolution model in Sect. 3; we present our results in Sect. 4; we discuss our findings in Sect. 5; we summarize our conclusions in Sect. 6.

\section{Observed evolution of the slow-rotator sequence from 120 Myr to $1 \mathrm{Gyr}$}

We constrain our models with the most up-to-date observations available for three rich well-studied clusters, namely the Pleiades, Praesepe, and NGC 6811 (Rebull et al. 2016; Douglas et al. 2019; Curtis et al. 2019, respectively). The slow-rotator sequence is clearly recognizable in the mass-rotation period diagrams of each of these clusters, between $M \approx 0.7$ and $1.3 M_{\odot}$ for the Pleiades, between $M \approx 0.3$ and $1.3 M_{\odot}$ for Praesepe, and between $M \approx 0.6$ and $1.3 M_{\odot}$ for NGC 6811 (see Fig. 1).

The Pleiades is one of the youngest clusters in which the slow-rotator sequence is observed; a large fraction of stars have not yet converged onto it. After approximately half a billion years, most of the stars of mass $\gtrsim 0.6 M_{\odot}$ in the Praesepe cluster are on the slow-rotator sequence. Finally, in NGC 6811, at the age of $1 \mathrm{Gyr}$, all stars with a measured rotation period are on the sequence.

In the following we adopt the ages of $120 \mathrm{Myr}, 700 \mathrm{Myr}$, and 1 Gyr for the Pleiades, Praesepe, and NGC 6811 clusters, respectively (see, e.g., Curtis et al. 2019). It should be noted that the age of Praesepe is a matter of some controversy in the literature. Douglas et al. (2019) calculated a gyrochronological age of 670 Myr for Praesepe, and discussed a compilation of isochronebased ages from the literature spanning the range 600-800 Myr. The oldest isochrone age $(800 \mathrm{Myr})$ is obtained when taking into account the effect of rotation on upper main sequence stars (Brandt \& Huang 2015, but see also Gossage et al. 2018). As noted above, in this work we fix the age of Praesepe at $700 \mathrm{Myr}$. The impact of the age uncertainty of this cluster on our fitting procedure is briefly discussed in Sect. 4.

As can be seen in Fig. 1, the slow-rotator sequences of Praesepe and NGC 6811 overlap below $M \lesssim 0.8 M_{\odot}$. This behavior is at odds with a simple description of the slow-rotator sequence as coherently evolving in the mass-rotation period diagram. Stars of mass $\approx 0.7 M_{\odot}$ seem to undergo a reduced spin-down epoch between $700 \mathrm{Myr}$ and $1 \mathrm{Gyr}$, while their more massive counterparts spin down as expected (Curtis et al. 2019). 


\section{Two-zone rotational evolution model with mass-dependent coupling}

\subsection{Description of the model}

We apply the rotational evolution model formulated by LS15 to describe the evolution of the slow-rotator sequence. The main assumption of the two-zone model is that the radiative zone and the convective envelope of the star are in a state of rigid rotation, with angular velocities $\Omega_{\mathrm{rad}}$ and $\Omega_{\mathrm{env}}$, respectively (MacGregor $\&$ Brenner 1991). These two quantities specify completely the rotational state of the star at a given time; the two zones have therefore angular momenta $J_{\text {rad }}=I_{\text {rad }} \Omega_{\text {rad }}$ and $J_{\text {env }}=I_{\text {env }} \Omega_{\text {env }}$, respectively, $I_{\text {rad }}$ and $I_{\text {env }}$ being their moments of inertia.

The physics included in the model is as follows:

1. Initial conditions. We assume initial rigid rotation, $\Omega_{\mathrm{rad}}\left(t_{0}\right)=\Omega_{\mathrm{env}}\left(t_{0}\right)$, with $t_{0} \approx 1 \mathrm{Myr}$. The initial period is assumed to be $P_{0}=8$ days for all masses; this choice is in qualitative agreement with the observed rotation period distribution of very young clusters (e.g., the 1-4 Myr Orion Nebula Cluster, Rebull 2001; see also Moraux et al. 2013). The interaction with the circumstellar disk is taken into account according to the disk-locking hypothesis (Koenigl 1991), i.e., assuming that $\Omega_{\text {env }}$ remains constant for the duration of the disk lifetime. We assume a circumstellar disk lifetime of $5 \mathrm{Myr}$, independent of mass (Hernández et al. 2008).

2. Wind braking. The overall rotational evolution is driven by the angular momentum loss from the surface, due to the torque imposed by the magnetized stellar wind (e.g., Schatzman 1962; Weber \& Davis 1967; Kawaler 1988). We adopt the wind braking law originally formulated by LS15 that incorporates the mass dependence proposed by Barnes \& Kim (2010), and follows the classical $\propto \Omega_{\mathrm{env}}^{3}$ rotation rate dependence of Kawaler (1988):

$\dot{J}_{\mathrm{wb}}=-K_{\mathrm{w}} K_{0}\left(\frac{I_{\mathrm{star}} \tau_{\mathrm{ov}}}{I_{\mathrm{star}, \odot} \tau_{\mathrm{ov}, \odot}}\right) \Omega_{\mathrm{env}}^{3}$.

In the equation above, $I_{\text {star }}=I_{\text {rad }}+I_{\text {env }}$ is the moment of inertia of the whole star, and $\tau_{\mathrm{ov}}$ is the convective overturn timescale of the convection zone. The product $I_{\mathrm{star}} \tau_{\mathrm{ov}}$ encompasses the mass dependence of our wind braking law; both quantities in the product are normalized to their values for a $1 M_{\odot}$ model, as indicated by the subscript " $\odot$ "; $K_{\mathrm{w}}$ is an overall calibration constant, whose scaling $K_{0}=1.11 \times 10^{47} \mathrm{~g} \mathrm{~cm}^{2} \mathrm{~s}$ depends on the choice of the units.

3. Internal angular momentum transport. As the envelope loses angular momentum, differential rotation develops, i.e., $\Omega_{\mathrm{rad}}(t) \gtrsim \Omega_{\mathrm{env}}(t)$; exchange of angular momentum between the two zones can also occur. During the pre-main sequence phase, the radiative interior grows at the expense of the convection zone, which comprises the whole star during the Hayashi phase (e.g., Kippenhahn et al. 2012). Once on the main sequence, angular momentum redistribution between the radiative and the convective zone can be mediated by several processes, whose relative importance is still an open issue (see Bouvier et al. 2014, for a recent review). Our model accounts for this effect phenomenologically by introducing a constant mass-dependent timescale $\tau_{\mathrm{c}}$ over which the excess of angular momentum of the interior, $\Delta J \equiv \frac{I_{\text {env }} J_{\text {rad }}-I_{\text {rad }} J_{\text {env }}}{I_{\text {rad }}+I_{\text {env }}}$, is transferred to the envelope. The rotational coupling timescale $\tau_{\mathrm{c}}$ is taken to be constant along the evolution, and to scale with the stellar mass as

$\tau_{\mathrm{c}}=\tau_{\mathrm{c}, \odot} \cdot\left(\frac{M_{*}}{M_{\odot}}\right)^{-\alpha}$.
This scaling, derived from semi-empirical fitting of LS15, was found by these authors to be robust to the choice of the wind braking law. It was also found to be in remarkably good agreement with the independent analysis of Somers \& Pinsonneault (2016).

The two-zone model equations for the evolution of $\Omega_{\text {rad }}$ and $\Omega_{\text {env }}$ are

$$
\begin{aligned}
& I_{\mathrm{rad}} \dot{\Omega}_{\mathrm{rad}}=+\frac{2}{3} \dot{M}_{\mathrm{rad}} R_{\mathrm{rad}}^{2} \Omega_{\mathrm{env}}-\frac{\Delta J}{\tau_{\mathrm{c}}}-\dot{I}_{\mathrm{rad}} \Omega_{\mathrm{rad}} \\
& I_{\mathrm{env}} \dot{\Omega}_{\mathrm{env}}=-\frac{2}{3} \dot{M}_{\mathrm{rad}} R_{\mathrm{rad}}^{2} \Omega_{\mathrm{env}}+\frac{\Delta J}{\tau_{\mathrm{c}}}-\dot{I}_{\mathrm{env}} \Omega_{\mathrm{env}}+\dot{J}_{\mathrm{wb}}
\end{aligned}
$$

where the dot denotes differentiation with respect to time; $R_{\text {rad }}$ and $M_{\text {rad }}$ are the radius and the mass of the radiative core, respectively. All the stellar structure parameters $\left(M_{\mathrm{rad}}, R_{\mathrm{rad}}, I_{\mathrm{rad}}, I_{\mathrm{env}}\right.$, $\left.\tau_{\mathrm{ov}}\right)$ are derived from stellar evolution models constructed with the Yale Rotational stellar Evolution Code (YREC) in its nonrotational configuration (Demarque et al. 2008).

Our model contains five parameters in total: the initial conditions are set by the initial rotation period $P_{0}$ and by the circumstellar disk lifetime $\tau_{\text {disk }}$; the wind braking law contains the calibration constant $K_{\mathrm{w}}$; the rotational coupling timescale is specified by $\tau_{\mathrm{c}, \odot}$ and $\alpha$.

\subsection{Comparison with LS15 and redetermining the model parameters}

Lanzafame \& Spada (2015) presented a comparison of several wind braking laws and a statistical determination of the parameters of the two-zone model $\left(P_{0}, \tau_{\text {disk }}, \tau_{\mathrm{c}}\right)$, based on the observational constraints on the slow-rotator sequence available at the time. The best-fitting values of the model parameters were obtained by means of a Markov chain Monte Carlo (MCMC) procedure for several different stellar masses in the range $M \approx$ $0.7-1.1 M_{\odot}$ (see LS15 for details). The main conclusions of their analysis can be summarized as follows. First, the wind braking law (1) (referred to as "KB" in LS15) captures the observed shape of the slow-rotator sequence sufficiently well that it can be used to describe the rotational evolution of stars of different masses with a unique value of the calibration constant $K_{\mathrm{w}}$. Second, moving from solar- to low-mass stars, it is increasingly important to take into account internal differential rotation in order to accurately reproduce the observations; the best results were obtained with a rotational coupling timescale that scales with stellar mass according to Eq. (2).

In this paper we adopt the two-zone model (3), and we retain the functional forms of the wind braking law (1) and of the massdependent coupling timescale (2). However, we redetermine the parameters to take into account the new data at lower masses available for Praesepe and NGC 6811. In particular, our goal is to ascertain whether our model can reproduce the stalled spindown of low-mass stars observed in the slow-rotator sequence of NGC 6811.

The identification of the slow-rotator sequence of clusters as young as the Pleiades is intrinsically uncertain and prone to subjective choices. The procedure adopted by LS15 was founded on noting that non-parametric fits to the slow-rotator sequences of clusters older than $\approx 0.5 \mathrm{Gyr}$ produce residuals that follow a quasi-Gaussian distribution. In order to trace the sequence back to younger cluster on the basis of a homogeneous criterion, LS15 chose the width of the slow-rotator sequence of younger clusters as the largest width that still produces a normal distribution of residuals with respect to the assumed average sequence. This 

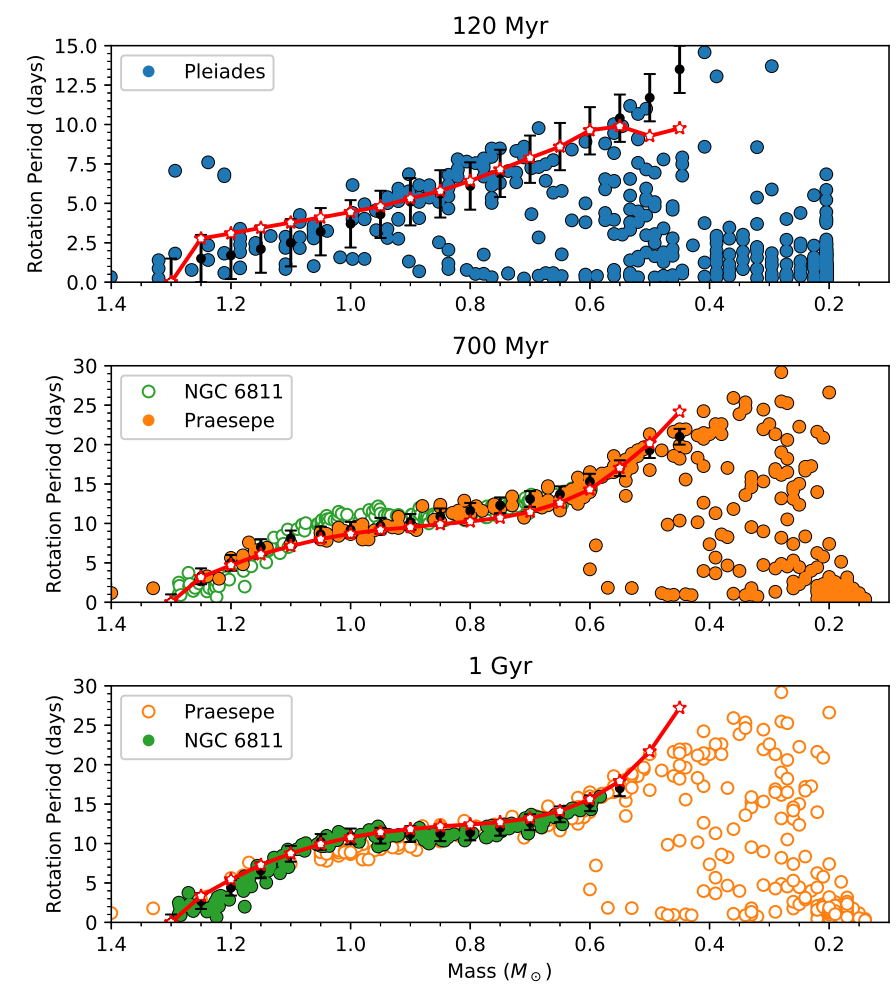

Fig. 2. Synthetic slow-rotator sequences predicted by our two-zone model at $120 \mathrm{Myr}, 700 \mathrm{Myr}$, and $1 \mathrm{Gyr}$ (red solid lines with stars) compared with the observations (colored circles). The black circles with error bars represent qualitative fits of the slow-rotator sequences; the error bars shown are 1.5 days for the Pleiades sequence and 1 day for Praesepe and NGC 6811.

procedure, however, cannot be applied to stars of mass below a certain threshold (see Sect. 3.2 and Fig. 2 of LS15). The lower limit of the slow-rotator sequence in the Pleiades found by LS15 is $\approx 0.75 M_{\odot}$. This implies that the rotational evolution of stars with mass lower than this limit cannot be constrained from the age of the Pleiades to the age of NGC 6811 using the LS15 definition.

In order to overcome this difficulty, in this work we adopt a width of the slow-rotator sequence of the Pleiades significantly larger than in LS15, which includes stars that are close to the sequence, but that may still not have fully converged to it. This allows us to put an approximate constraint at $\approx 120 \mathrm{Myr}$ to the subsequent rotational evolution of stars with mass as low as $M \approx$ $0.4 M_{\odot}$. This more relaxed criterion (in comparison with LS15) in defining the slow-rotator sequence of the Pleiades, however, results in lower rotation periods used in the fit at $120 \mathrm{Myr}$ in the mass range $M \lesssim 0.9 M_{\odot}$. This could be the cause of the small bias observed at later ages (see Fig. 2).

In LS15 the parameters governing the initial conditions, $P_{0}$ and $\tau_{\text {disk }}$, were determined by the requirement to reproduce the slow-rotator sequence of the Pleiades. In this work, we have adjusted the values of $P_{0}$ and $\tau_{\text {disk }}$ to reproduce the approximate slow-rotator sequence of the Pleiades, as shown in Fig. 2. The values adopted do not differ significantly from those of LS15.

The parameters $K_{\mathrm{w}}, \tau_{\mathrm{c}, \odot}$, and $\alpha$, on the other hand, control the subsequent evolution of the slow-rotator sequence from $\approx 100 \mathrm{Myr}$ onward. These parameters are strongly correlated with each other (see Lanzafame \& Spada 2015), and must therefore be re-evaluated together. The new data on Praesepe and NGC 6811, extending significantly the mass range sampled,

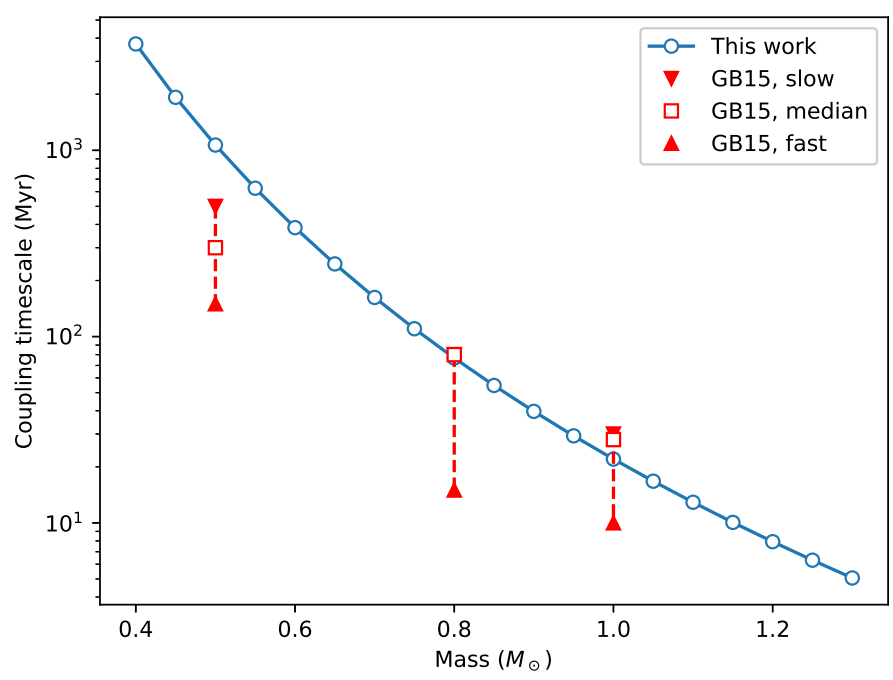

Fig. 3. Comparison of the best-fitting rotational coupling timescale found in this work and in Gallet \& Bouvier (2015).

provide the opportunity to refine the estimate of these parameters, in particular the power law exponent $\alpha$.

The revised best-fitting values of the parameters $K_{\mathrm{w}}, \tau_{\mathrm{c}, \odot}$, and $\alpha$ were obtained using a Python implementation of the Nelder \& Mead (1965) simplex algorithm, which is available in the function optimize . minimize, which is part of the SciPy ${ }^{1}$ package. Our revised estimates are

$K_{\mathrm{W}}=3.4 ; \quad \tau_{\mathrm{c}, \odot}=22 \mathrm{Myr} ; \quad \alpha=5.6$.

It should be noted that these parameters were not obtained by LS15 as a direct result of their MCMC procedure. Rather, $K_{\mathrm{w}}^{\mathrm{LS} 15}=4.5$ was obtained as an average over the entire mass range considered $\left(\approx 0.7-1.1 M_{\odot}\right)$, while $\tau_{\mathrm{c}, \odot}$ and $\alpha$ were determined a posteriori from the values of $\tau_{\mathrm{c}}$ fitted independently at each mass. Our current estimate of $K_{\mathrm{w}}$ is compatible with the LS15 value within the uncertainties, and is in excellent agreement with their estimate for a $1 M_{\odot}$ star (3.6, cf. Table 3 in LS15). The value of $\tau_{\mathrm{c}, \odot}$ is nearly unchanged $\left(\tau_{\mathrm{c}, \odot}^{\mathrm{LS} 15}=25 \mathrm{Myr}\right)$. Finally, the recalibrated $\alpha$ is significantly different from $\alpha^{\mathrm{LS} 15}=7.3$.

This difference is mainly due to the extension to lower masses, which in principle puts stronger constraints on the $\tau_{\mathrm{c}}$ versus mass relationship. There are, however, other issues that should be taken into account, namely the relationship may not be accurately reproduced by a simple power law, the scatter at $M \lesssim 0.9 M_{\odot}$ in LS15 may be underestimated due to limited sampling, or the extrapolation of the slow-rotator sequence of the Pleiades to $M<0.8 M_{\odot}$ adopted in this work may not be appropriate for an accurate estimate of $\alpha$. Furthermore, the different fitting strategy, together with the correlations among the parameters, could also play a role.

A comparison with the estimates of $\tau_{\mathrm{c}}$ at $M=1.0,0.8$, and $0.5 M_{\odot}$ from Gallet \& Bouvier (2015) (see Fig. 3) confirms the steepness of the $\tau_{\mathrm{c}}$ versus mass relation, although it suggests a somewhat smaller slope than the one derived in the present work. It should be noted, however, that Gallet \& Bouvier (2015) did not attempt to identify and fit a slow-rotator sequence, but rather adopted the 25th and 95th percentiles as representative of slow and fast rotators, respectively. As a consequence, the representative periods for their slow-rotator sample tend to be lower than those estimated in this work (and in LS15) for the slow-rotator

1 http://www.scipy.org/ 

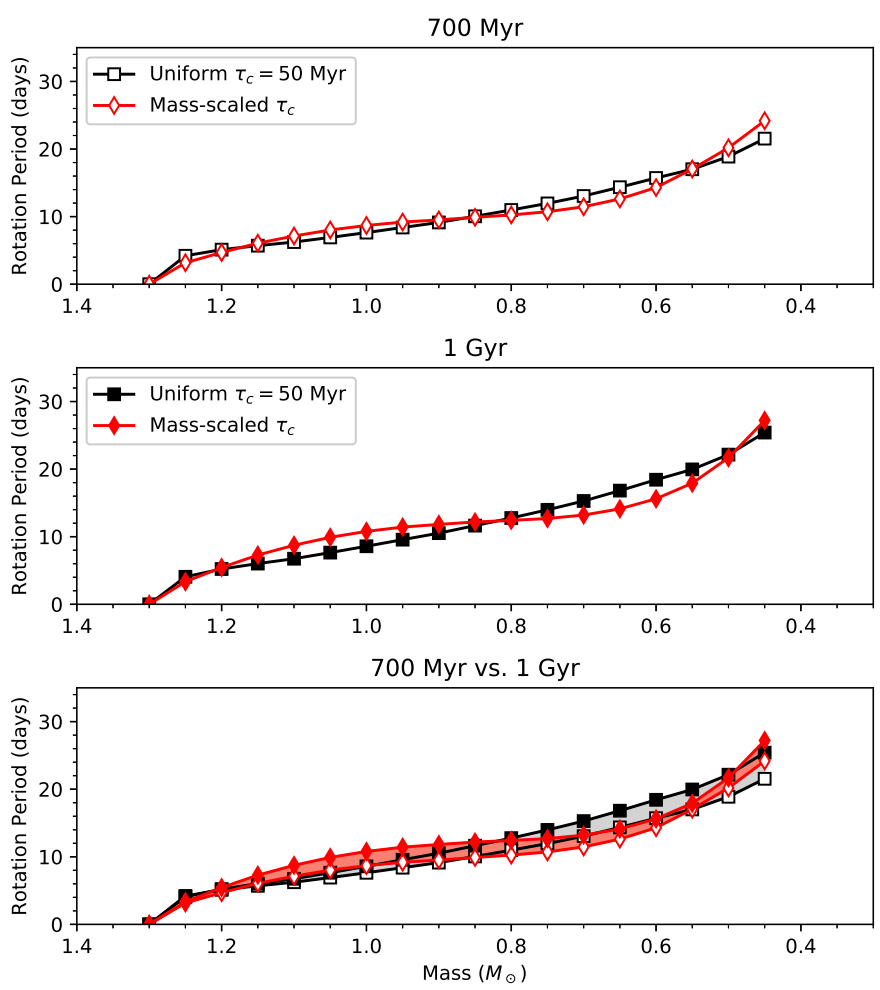

Fig. 4. Synthetic slow-rotator sequences obtained with uniform $\tau_{\mathrm{c}}$ vs. mass-dependent $\tau_{\mathrm{c}}$. Bottom panel: comparison of the difference between the slow-rotator sequences at $700 \mathrm{Myr}$ and $1 \mathrm{Gyr}$ (gray and red shading for the uniform $\tau_{\mathrm{c}}$ and the mass-dependent $\tau_{\mathrm{c}}$, respectively).

sequence. Since the time required to converge to the slow-rotator sequence increases with decreasing mass, the difference in the adopted periods increases at lower masses, which explains the somewhat weaker $\tau_{\mathrm{c}}$ versus mass relation found by Gallet \& Bouvier (2015).

\section{Results}

\subsection{Modeling the evolution of the slow-rotator sequence}

The results of our best-fitting two-zone model are compared with the observations in Fig. 2. A qualitative fit of the slow-rotator sequence is also shown for each cluster (black circles with error bars). For the purposes of the present study, we assume a dispersion of the slow-rotator sequence of 1.5 days for the Pleiades, and of 1.0 days for Praesepe and NGC 6811. These dispersions, represented by the error bars in Fig. 2, are significantly larger than those obtained from the non-parametric fits of LS15 (cf. their Table 2: $\sigma^{\mathrm{LS} 15}=0.35$ days, 0.63 days, and 0.46 days for the Pleiades, Praesepe, and NGC 6811, respectively). As discussed in Sect. 3.2, this somewhat more relaxed definition of the slowrotator sequence was necessary to extend it to low-mass stars $\left(M<0.7 M_{\odot}\right)$, to which the more rigorous criterion adopted by LS15, based on the quasi-Gaussian distribution of the residuals around the average sequence, cannot be applied.

The overall fit of the slow-rotator sequences of the Pleiades, Praesepe, and NGC 6811 shown in Fig. 2 is satisfactory. In particular, our model predicts a reduced spin-down for stars in the mass range $0.6-0.8 M_{\odot}$ between $700 \mathrm{Myr}$ and $1 \mathrm{Gyr}$. This effect, combined with the intrinsic scatter of the slow-rotator sequences of Praesepe and NGC 6811, leads to an apparent superposition

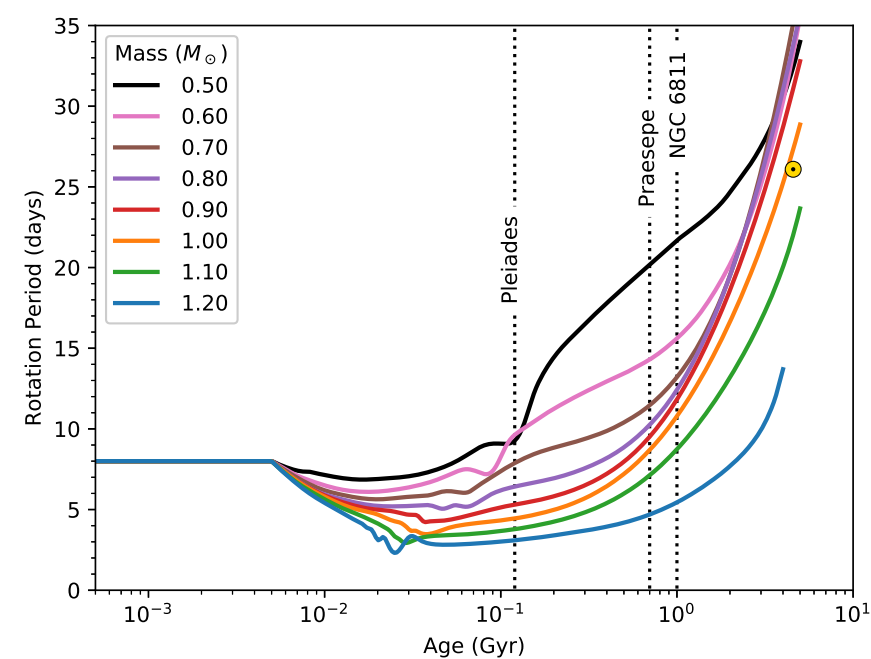

Fig. 5. Rotational evolution tracks constructed with our two-zone model. The vertical dotted lines indicate the ages of the clusters used for comparison (cf. Fig. 2); the Sun is also plotted for reference.

of the sequences in the mass-rotation period diagram, and thus in turn to an apparent stalling of the spin-down of these stars.

The key ingredient in reproducing the reduced spin-down is the scaling of the rotational coupling timescale with the mass of the star according to Eq. (2). This is immediately apparent when we compare the predictions of our reference model with those obtained assuming a value of $\tau_{\mathrm{c}}$ independent of stellar mass (all the other parameters being the same).

As Fig. 4 shows, the uniform- $\tau_{\mathrm{c}}$ assumption produces a slowrotator sequence with a steeper slope at the age of NGC 6811 in the mass range $\approx 0.5-0.85 \mathrm{M}_{\odot}$. Moreover, the difference between the slow-rotator sequences at $700 \mathrm{Myr}$ and at $1 \mathrm{Gyr}$ (bottom panel of the figure) monotonically increases toward lower masses for the uniform- $\tau_{\mathrm{c}}$ model. We conclude that a model with $\tau_{c}$ independent of mass cannot achieve a satisfactory fit of the slow-rotator sequences of Praesepe and NGC 6811. Such a model, in particular, fails to reproduce the stalled spindown of the $\approx 0.7 M_{\odot}$ stars.

On the contrary, the two-zone model implementing the scaling of $\tau_{\mathrm{c}}$ according to Eq. (2) produces a separation between the slow-rotator sequences at $700 \mathrm{Myr}$ and $1 \mathrm{Gyr}$ that is largest at $\approx 1 M_{\odot}$ and decreases at lower masses, thus correctly reproducing the merging of the slow-rotator sequences observed in Praesepe and NGC 6811.

Given the significant uncertainty in the age of Praesepe, we tested the effect of varying it within the range $600-800 \mathrm{Myr}$ (Douglas et al. 2019). Using an age of $600 \mathrm{Myr}$, the fit of the sequence of Praesepe is significantly worsened compared to our adopted age of $700 \mathrm{Myr}$. On the contrary, the fit is improved when using $800 \mathrm{Myr}$, bringing it to the same level of agreement as for the Pleiades and NGC 6811.

Figure 5 shows the evolution of the surface rotation period calculated with our rotational evolution model. This alternative view further illustrates the effect of the rotational coupling. Stars of mass $\lesssim 0.7 M_{\odot}$ experience a significantly reduced surface spindown than their more massive counterparts between $700 \mathrm{Myr}$ and $1 \mathrm{Gyr}$. This effect is the direct consequence of the redistribution of angular momentum from the radiative zone to the convective envelope, which temporarily offsets the angular momentum loss at the surface. 


\subsection{Wind braking vs. rotational coupling}

The rotational evolution according to the two-zone model Eq. (3) is controlled by the wind braking, described by Eq. (1), and by the rotational coupling, characterized by Eq. (2). Which of these two processes dominates over the other depends on the mass of the star and on its age.

We can gain some insight into the relative importance of these two effects by comparing their timescales. The wind braking law (1) has a steep dependence on the surface rotation rate $\left(\propto \Omega_{\text {env }}^{3}\right)$. The efficiency of the angular momentum loss therefore varies by several orders of magnitude between the zero age main sequence, when the rotation rate is highest for all masses, and the mature main sequence phases ( $\gtrsim 1 \mathrm{Gyr})$. We can characterize the wind braking efficiency by the timescale:

$\frac{1}{\tau_{\mathrm{wb}}} \equiv \frac{\dot{J}_{\mathrm{wb}}}{J_{\mathrm{env}}}$

The rotational coupling timescale, on the other hand, is assumed to be constant for the entire evolution of the star.

The ratio $\tau_{\mathrm{wb}} / \tau_{\mathrm{c}}$ is a measure of the relative importance of the angular momentum loss and the internal angular momentum transport in the overall angular momentum balance of the star. This ratio is plotted in Fig. 6. The early pre-main sequence rotational evolution corresponds to a regime in which the star rotates as a solid body, and $\tau_{\mathrm{wb}} / \tau_{\mathrm{c}} \gg 1$. As the star spins up and a radiative core develops, it enters a regime (marked by the gray shading in Fig. 6) in which $\tau_{\mathrm{wb}} / \tau_{\mathrm{c}}<1$. The magnetic braking, which scales as $\sim \Omega_{\mathrm{env}}^{3}$, reaches its maximum efficiency at the end of the pre-main sequence spin-up phase, and the angular momentum transport from the interior cannot compensate for the loss at the surface. As a result, a significant differential rotation develops and maintains during the early main sequence. After a mass-dependent time interval, as dictated by Eq. (2), the star re-enters the opposite regime, i.e., $\tau_{\mathrm{wb}} / \tau_{\mathrm{c}}>1$. In this regime the core-envelope coupling is more efficient, and eventually brings the star to a quasi solid-body rotation. Close to the transition between the two regimes (i.e., when $\tau_{\mathrm{wb}} / \tau_{\mathrm{c}} \approx 1$ ) the transport of angular momentum from the interior to the surface reduces the spin-down observed at the surface with respect to the wind braking as if it were acting alone. Reduced, or stalled, spin-down can be expected at this stage. When a quasi solid-body regime is established, the stellar spin-down is essentially controlled by the magnetic braking alone.

For stars of solar mass or higher, the rotational coupling efficiency catches up with the wind braking already by the age of the Pleiades, and differential rotation has essentially no chance to develop at later times. Conversely, the rotational coupling efficiency can compete with wind braking at $\gtrsim 700 \mathrm{Myr}$ for stars of mass $\approx 0.75 M_{\odot}$, and only after several Gyr for those of mass $\approx 0.5 M_{\odot}$ (see also LS15). At the age of Praesepe, the stars in the mass range $0.6-0.8 M_{\odot}$ have either just crossed or are about to cross the condition $\tau_{\mathrm{wb}} / \tau_{\mathrm{c}} \approx 1$, and therefore their surface rotation is significantly affected by the angular momentum transport from the interior to the surface. A quasi solidbody rotation regime, in which the surface rotation is dominated by the wind braking, will be established only at a later time. For instance, a star of mass $0.8 M_{\odot}$ attains this conditions at $\approx 2-3$ Gyr.

\section{Discussion}

We presented a simple model for the rotational evolution of solar-like stars that contains two main physical ingredients: the

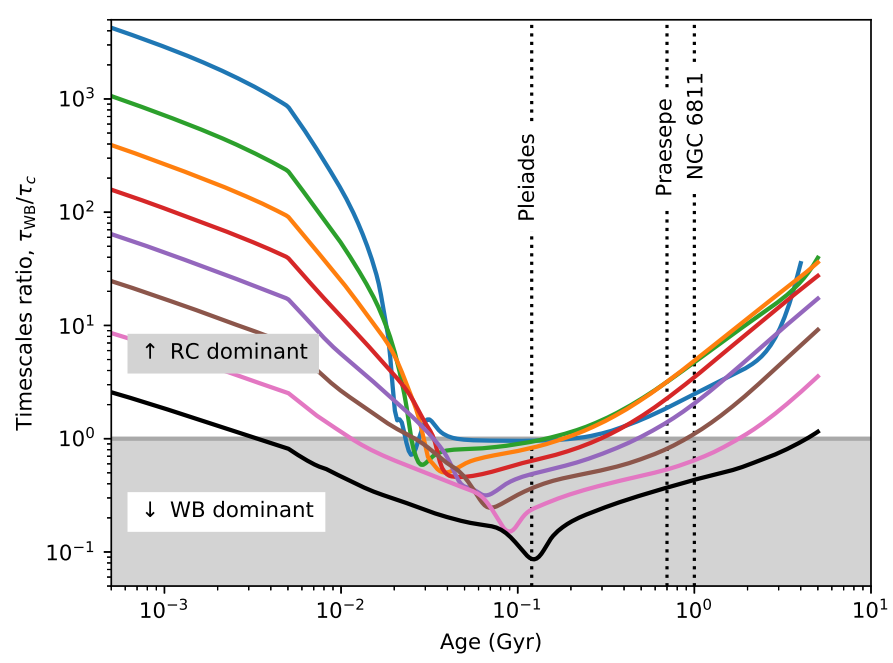

Fig. 6. Ratio of the wind braking timescale $\tau_{\mathrm{wb}}$ (Eq. (5)) to the rotational coupling timescale $\tau_{\mathrm{c}}$ for the same tracks shown in Fig. 5. The shaded area of the plot indicates the evolutionary phases during which wind braking $(\mathrm{WB})$ is dominant over the rotational coupling (RC; see discussion in the text).

magnetized wind braking at the surface, and the angular momentum transport (or coupling) between the interior and the surface.

The essential role of the rotational de-coupling and recoupling between the radiative interior and the convective envelope in the pre-main sequence and early main sequence evolution of solar-like stars has been recognized since the works of Endal \& Sofia (1981), Stauffer et al. (1984, 1985), Pinsonneault et al. (1989), and Soderblom et al. (1993), just to name a few. This effect has been incorporated phenomenologically in all subsequent modeling efforts, and several candidate processes have been proposed (MHD waves/instabilities, gravity waves; see, e.g., Charbonneau \& MacGregor 1993; Ruediger \& Kitchatinov 1996; Spruit 2002; Talon \& Charbonnel 2003; Charbonnel \& Talon 2005; Spada et al. 2010; Brun et al. 2011; Oglethorpe \& Garaud 2013). In spite of this, the physical nature of the processes redistributing angular momentum in the interior of stars is still a major open question.

Previous works (e.g., Denissenkov et al. 2010; Gallet \& Bouvier 2015) have underlined the strong mass dependence of the core-envelope rotational coupling timescale. By concentrating on the rotational evolution of the slow-rotator sequence (or "I-sequence" in the terminology of Barnes 2003), LS15 quantified the mass dependence of the rotational coupling timescale. The model proposed by LS15, which incorporates this dependence, can reproduce the evolution of the slow-rotator sequence accurately.

The new rotational data for NGC 6811 (Curtis et al. 2019) provide previously unavailable constraints by extending the range of mass with known rotation periods at $1 \mathrm{Gyr}$ down to $M \approx 0.6 M_{\odot}$. Together with the new Praesepe data (Douglas et al. 2019), these prompted us to re-evaluate the parameters of the LS15 model.

The recalibrated parameters are consistent with the results of LS15 within the uncertainties of their fit to the data available at that time. Our updated model also explains quantitatively the apparent halt in the spin-down of low-mass stars $\left(M \approx 0.7 M_{\odot}\right)$ between $700 \mathrm{Myr}$ and $1 \mathrm{Gyr}$, recently discovered by Curtis et al. (2019). This phenomenon is a manifestation of the angular momentum redistribution from the interior of the star to its outer envelope, which can temporarily compensate for the angular momentum lost from the surface via magnetic braking. 
It should be emphasized that the observed lack of spin-down arises naturally in our model from simple assumptions regarding the mass dependence of the angular momentum coupling timescale. In other words, it is not necessary to postulate a phase of weakened wind braking regime to explain the evolution of the slow-rotator sequence as observed in open clusters. In addition, our model provides testable predictions on the duration of this reduced spin-down phase, and on the epoch at which stars of different mass are expected to experience it.

Barnes (2003) originally defined the I-sequence observed in open clusters as comprising stars spinning down according to the phenomenological relation $P_{\text {rot }} \propto \sqrt{t}$ (Skumanich 1972). Our results show that such a working definition still retains its approximate validity even in the light of the most recent observations. The overall shape of the slow-rotator sequence is captured remarkably well by the wind braking law (1), which couples the mass dependence proposed by Barnes \& Kim (2010) with a dependence on $\Omega_{\mathrm{env}}^{3}$, which is known to reproduce the Skumanich (1972) law (see, e.g., Kawaler 1988).

A comparison of the rotational evolution in the mass range $0.85-1.10 M_{\odot}$ according to different wind braking laws is shown in Fig. 6 in LS15. The wind braking laws considered there (Matt et al. 2015; Gallet \& Bouvier 2015, and the original suggestion of LS15, based on the mass dependence of Barnes 2010) were found to be in remarkable agreement, once the freely adjustable parameters are calibrated. The main difference among these wind braking prescriptions is a variable amount of residual mass dependence in the adjustable parameters (mainly $K_{\mathrm{w}}$ ), suggesting that they recover the mass dependence of the slow-rotator sequence observed in open clusters with different degrees of success (see LS15 for details). On the other hand, the rotational coupling timescale and its mass dependence, which are the focus of this work, are remarkably independent of the wind braking law (see Fig. 5 in LS15). For these reasons, we did not repeat the comparison with other wind braking laws in this paper.

Departures from the Skumanich (1972) law in the slowrotator sequence have been known for a long time (e.g., Meibom et al. 2009, 2011), and were already interpreted by LS15 as arising from the re-coupling of the surface with the interior. This effect is the manifestation of a strong dependence of the coupling timescale on stellar mass (cf. Eq. (2)). Building on our previous results, we show that the halt in the spin-down of low-mass stars reported by Curtis et al. (2019) is a consequence of the re-coupling occurring at the age of $\lesssim 1 \mathrm{Gyr}$ for $\approx 0.7 M_{\odot}$ stars.

The new observations of Curtis et al. (2019) allow us to refine the determination of the parameters in Eq. (2), to extend its validity to lower masses. This places valuable constraints on the unknown physical nature of the processes that transport angular momentum in the interior of solar-like main sequence stars. In particular, we confirm (within the uncertainties) the steep mass dependence found by LS15, and independently recovered by Somers \& Pinsonneault (2016). A successful theoretical description of the rotational coupling in solar-like stars from first principles should be able to explain the semi-empirical result of Eq. (2).

An implicit assumption of the treatment of the internal rotational coupling in the two-zone model is that the coupling timescale $\tau_{\mathrm{c}}$ is constant in time. This is of course a simplification, as a number of different processes, each with its own timescale and dependence on the stellar structure parameters, may in principle contribute to the transport of angular momentum in stellar interiors (hydrodynamical instabilities, e.g., Pinsonneault et al. 1989; MHD instabilities, e.g., Charbonneau

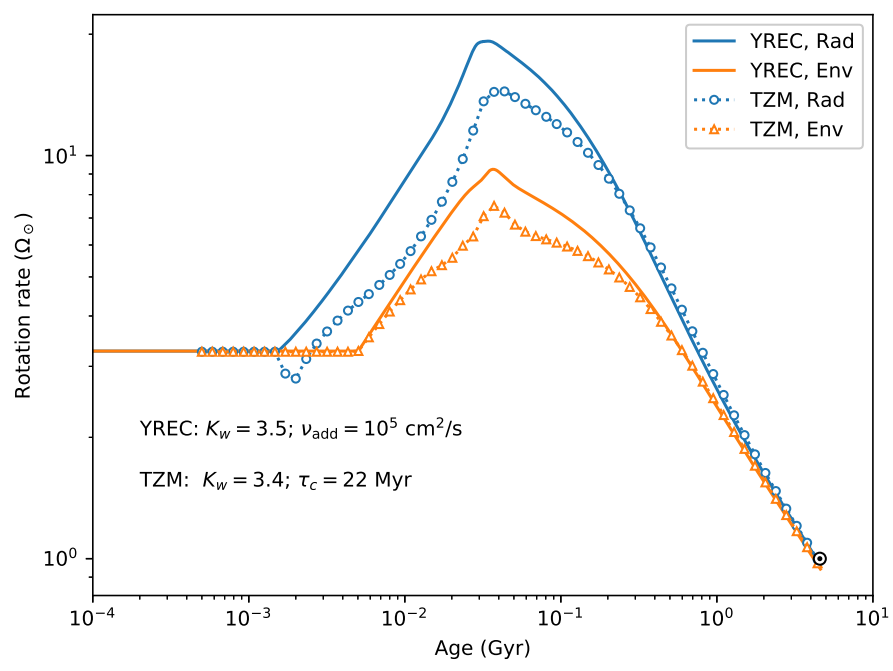

Fig. 7. Rotational evolution of a $1 M_{\odot}$ star from birth line to solar age calculated with the YREC code in its rotational configuration (Spada et al. 2016) compared with the results of the two-zone model (TZM) for the same mass.

\& MacGregor 1993; Ruediger \& Kitchatinov 1996; internal gravity waves, e.g., Talon \& Charbonnel 2003; Charbonnel \& Talon 2005). It should be noted, however, that after complete rotational coupling of the stellar interior with the surface is established (at a mass-dependent age), the parameter $\tau_{\mathrm{c}}$ becomes irrelevant, since the star remains fully coupled for the rest of its main sequence evolution. This approximation is therefore sufficient to constrain the timescale associated with the angular momentum redistribution process that, integrated over the early main sequence lifetime, operates most efficiently. We note in passing that although the assumption of a rotational coupling timescale constant in time is adequate to represent our current level of understanding of this process for main sequence stars, it is inconsistent with the observed rotational evolution of subgiants and red giant stars (e.g., Spada et al. 2016; Eggenberger et al. 2019).

The next step in order of complexity in modeling the rotational evolution of solar-like stars is to incorporate the prescriptions for wind braking and angular momentum transport (e.g., Eqs. (1) and (2)) in a stellar evolution code. This approach is more self-consistent than the two-zone model, as it allows us to take into account the mutual effects of rotation and stellar structure (Endal \& Sofia 1976); moreover, it provides information on the detailed rotational profile inside the star, as opposed to the integrated quantity $\left(\Omega_{\mathrm{rad}}-\Omega_{\mathrm{env}}\right)$.

In Fig. 7 we compare the two-zone model predictions for a $1 M_{\odot}$ star with an evolutionary calculation constructed with YREC in its rotational configuration. The YREC run implements disk-locking (on a timescale of $5 \mathrm{Myr}$ ), the original Kawaler (1988) wind braking law without saturation (which for a $1 M_{\odot}$ star is indistinguishable from Eq. (1)), and diffusive transport of angular momentum in the interior with a constant diffusion coefficient $v_{\text {add }}$. We note that a fairly straightforward correspondence can be established between the quantities $\nu_{\text {add }}$ and $\tau_{\mathrm{c}}$ (see Denissenkov et al. 2010). The calibration constant $K_{\mathrm{w}}$ and the diffusion coefficient $v_{\text {add }}$ were adjusted to obtain an evolution consistent with the solar constraints at $4.57 \mathrm{Gyr}$ (cf. the solar benchmark calculation in Sect. 3.2 of Spada et al. 2016). The results of YREC and of the two-zone model differ moderately during the pre-main sequence and early main sequence phases, 
which are characterized by the most rapid structural changes. The two modeling approaches give essentially identical results at ages later than $\approx 300 \mathrm{Myr}$.

The deviations from a purely (Skumanich 1972) spin-down that depend on the stellar mass are of practical importance for gyrochronology. The original gyrochronological relations, based on the assumption of a purely factorable color and age dependence (where the color is used as a proxy of stellar mass), $P_{\text {rot }}=$ $f(B-V) g(t)$, were already shown to be untenable by Barnes $\&$ Kim (2010). Our model is a promising step toward the construction of more accurate gyrochronology relations, extracted from a simple physical model. This improvement comes at the price of not having a simple all-encompassing formula that can be readily inverted to derive the age from the rotation period. For convenience, we provide updated rotational isochrones for a wide range of ages in Appendix A.

Our model reproduces satisfactorily the slow-rotator sequence, as observed in the Pleiades cluster $(\approx 100 \mathrm{Myr})$, and its subsequent evolution, as constrained by the recent observations of Praesepe and NGC 6811 (700 Myr and $1 \mathrm{Gyr}$, respectively), in the solar-like stars regime $\left(\approx 0.4-1.3 M_{\odot}\right)$. A two-zone model like ours, however, is obviously not applicable without modifications to fully convective stars $\left(\lesssim 0.35 M_{\odot}\right)$. Whether such lowmass stars converge on the slow-rotator sequence and whether their evolution is similar to that of their more massive counterparts in spite of this qualitative difference in their interior structure are still open questions.

\section{Conclusions}

We presented a model for the rotational evolution of solar-like stars that reproduces the features of the slow-rotator sequence observed in open clusters between $\approx 100$ and 1000 Myr. In particular, our model captures satisfactorily the mass dependence of the slow-rotator sequence in the range $0.4-1.3 M_{\odot}$, and the reduced spin-down observed in stars of mass $\lesssim 0.8 M_{\odot}$ between $700 \mathrm{Myr}$ and $1 \mathrm{Gyr}$.

The key ingredients of the model are the scalings of the wind braking law and of the rotational coupling timescale with stellar mass. The former is nicely represented by the product of the moment of inertia of the star times its convective overturn timescale; the latter follows a steep power law of exponent $\approx-5.6$, with a scaling coefficient corresponding to a coupling timescale of $\approx 22 \mathrm{Myr}$ for a $1 M_{\odot}$ star.

Our results are a promising step toward more physically motivated gyrochronology relations, and highlight the necessity of taking into account the internal transport of angular momentum in modeling the rotational evolution of solar-like stars. In addition, our model provides constraints on the currently unknown processes that transport angular momentum in the interior of solar-like stars.
Acknowledgements. FS is supported by the German space agency (Deutsches Zentrum für Luft- und Raumfahrt) under PLATO Data Center Grant 50001501.

\section{References}

Barnes, S. A. 2003, ApJ, 586, 464

Barnes, S. A. 2007, ApJ, 669, 1167

Barnes, S. A. 2010, ApJ, 722, 222

Barnes, S. A., \& Kim, Y.-C. 2010, ApJ, 721, 675

Barnes, S. A., Spada, F., \& Weingrill, J. 2016a, Astron. Nachr., 337, 810

Barnes, S. A., Weingrill, J., Fritzewski, D., et al. 2016b, ApJ, 823, 16

Bouvier, J., Matt, S. P., Mohanty, S., et al. 2014, Protostars Planets VI (Tucson: University of Arizona Press), 433

Brandt, T. D., \& Huang, C. X. 2015, ApJ, 807, 24

Brown, T. M. 2014, ApJ, 789, 101

Brun, A. S., Miesch, M. S., \& Toomre, J. 2011, ApJ, 742, 79

Charbonneau, P., \& MacGregor, K. B. 1993, ApJ, 417, 762

Charbonnel, C., \& Talon, S. 2005, Science, 309, 2189

Curtis, J. L., Agüeros, M. A., Douglas, S. T., et al. 2019, ApJ, 879, 49

Demarque, P. R., \& Larson, R. B. 1964, ApJ, 140, 544

Demarque, P., Guenther, D. B., Li, L. H., et al. 2008, Ap\&SS, 316, 31

Denissenkov, P. A., Pinsonneault, M., Terndrup, D. M., et al. 2010, ApJ, 716, 1269

Douglas, S. T., Curtis, J. L., Agüeros, M. A., et al. 2019, ApJ, 879, 100 Eggenberger, P., Buldgen, G., \& Salmon, S. J. A. J. 2019, A\&A, 626, L1 Endal, A. S., \& Sofia, S. 1976, ApJ, 210, 184

Endal, A. S., \& Sofia, S. 1981, ApJ, 243, 625

Gallet, F., \& Bouvier, J. 2015, A\&A, 577, A98

Gondoin, P. 2017, A\&A, 599, A122

Gossage, S., Conroy, C., Dotter, A., et al. 2018, ApJ, 863, 67

Hernández, J., Hartmann, L., Calvet, N., et al. 2008, ApJ, 686, 1195

Janes, K., Barnes, S. A., Meibom, S., et al. 2013, AJ, 145, 7

Kawaler, S. D. 1988, ApJ, 333, 236

Koenigl, A. 1991, ApJ, 370, L39

Kippenhahn, R., Weigert, A., \& Weiss, A. 2012, Stellar Structure and Evolution, 2nd edn. (Berlin, Heidelberg: Springer-Verlag)

Kraft, R. P. 1967, ApJ, 150, 551

Lanzafame, A. C., \& Spada, F. 2015, A\&A, 584, A30

MacGregor, K. B., \& Brenner, M. 1991, ApJ, 376, 204

Matt, S. P., Brun, A. S., Baraffe, I., et al. 2015, ApJ, 799, L23

Meibom, S., Mathieu, R. D., \& Stassun, K. G. 2009, ApJ, 695, 679

Meibom, S., Mathieu, R. D., Stassun, K. G., et al. 2011, ApJ, 733, 115

Moraux, E., Artemenko, S., Bouvier, J., et al. 2013, A\&A, 560, A13

Nelder, J. A., \& Mead, R. 1965, Comput. J., 7, 308

Newton, E. R., Mondrik, N., Irwin, J., et al. 2018, AJ, 156, 217

Oglethorpe, R. L. F., \& Garaud, P. 2013, ApJ, 778, 166

Pinsonneault, M. H., Kawaler, S. D., Sofia, S., et al. 1989, ApJ, 338, 424

Rebull, L. M. 2001, AJ, 121, 1676

Rebull, L. M., Stauffer, J. R., Bouvier, J., et al. 2016, AJ, 152, 113

Ruediger, G., \& Kitchatinov, L. L. 1996, ApJ, 466, 1078

Schatzman, E. 1962, Ann. Astrophys., 25, 18

Skumanich, A. 1972, ApJ, 171, 565

Soderblom, D. R., Stauffer, J. R., Hudon, J. D., et al. 1993, ApJS, 85, 315

Somers, G., \& Pinsonneault, M. H. 2016, ApJ, 829, 32

Spada, F., Lanzafame, A. C., \& Lanza, A. F. 2010, MNRAS, 404, 641

Spada, F., Gellert, M., Arlt, R., et al. 2016, A\&A, 589, A23

Spada, F., Demarque, P., Kim, Y.-C., et al. 2017, ApJ, 838, 161

Spruit, H. C. 2002, A\&A, 381, 923

Stauffer, J. R., Hartmann, L., Soderblom, D. R., et al. 1984, ApJ, 280, 202

Stauffer, J. R., Hartmann, L. W., Burnham, J. N., et al. 1985, ApJ, 289, 247

Talon, S., \& Charbonnel, C. 2003, A\&A, 405, 1025

Weber, E. J., \& Davis, L. 1967, ApJ, 148, 217 


\section{Appendix A: Rotational isochrones}

Selected rotational isochrones calculated with our updated twozone model are reported in Table A.1, and are plotted in Fig. A.1. The table lists the surface rotation period, in days, as a function of stellar mass and age; $(B-V)$ colors are also given, as calculated from a solar metallicity $400 \mathrm{Myr}$ isochrone from the YaPSI database (Spada et al. 2017).

In Fig. A. 2 we compare our current isochrones with those of LS15. The updated isochrones are consistent with our previous results in the range of overlap. A moderate disagreement is visible at ages $\gtrsim 2 \mathrm{Gyr}$, reflecting the different choices of the overall calibration constant $K_{\mathrm{w}}$ in Eq. (1).

Table A.1. Rotational isochrones constructed with our updated two-zone model.

\begin{tabular}{|c|c|c|c|c|c|c|c|c|c|c|c|c|c|c|c|c|c|c|}
\hline \multirow[t]{2}{*}{ Mass } & \multirow[t]{2}{*}{$(B-V)$} & \multicolumn{17}{|c|}{ Ages (Gyr) } \\
\hline & & 10 & 12 & 15 & 0.20 & 0.22 & 25 & .30 & 0.40 & 0.50 & 0.60 & 0.70 & 1.00 & 1.50 & 2.00 & .50 & .00 & 4.57 \\
\hline 0.45 & 477 & ) & 6 & 4 & 14.87 & 5 & 0 & 4 & 9 & 4 & 3 & 18 & 21 & 38 & .11 & .90 & .36 & 4.4 \\
\hline & & 08 & 27 & .81 & 14.43 & & & 49 & 17.80 & 76 & & 20.18 & 21.68 & 3.19 & 4.66 & 26.11 & 0.52 & 32.41 \\
\hline & & 8.40 & 9.89 & 11.53 & 12.43 & 12.76 & .24 & 14.00 & 15.30 & 16.18 & & 17.04 & 17.92 & 19.47 & 21.26 & 23.27 & .82 & 32.72 \\
\hline 60 & & 26 & 9.63 & .38 & 11.24 & 11.50 & 1.84 & 12.28 & 12.94 & 13.43 & 13 & 14.30 & 15.61 & 17.87 & 20.20 & 22.62 & 30.29 & 33.27 \\
\hline & & 18 & 60 & & & & .39 & 10.71 & 11.22 & & & & 12 & 77 & 2 & 2.62 & 49 & 34.70 \\
\hline & & 38 & 88 & 8.38 & 83 & 8.9 & 9.12 & 9.38 & 9.87 & 10.40 & & 11.46 & 13.21 & 16.30 & 19.50 & 22.77 & 96 & 35.11 \\
\hline & & 6.73 & .15 & 7.51 & 7.87 & 7.97 & 8.1 & 8.39 & 8.92 & 9.49 & 10.10 & 10.72 & 12.69 & 16.12 & 19.55 & 22.86 & 31.74 & 34.68 \\
\hline & & 9 & 42 & 6.6 & 6 & 7.0 & & 7.56 & 8.1 & 8.8 & & 10.25 & 12.42 & 16 & 9 & 22.69 & 1 & 33.76 \\
\hline & & & 9 & & & & 6. & & 7.6 & 0. & & 9. & 12.18 & 5 & 19 & 22.26 & 1 & 32.56 \\
\hline & & 14 & 31 & 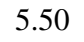 & 82 & 5. & 6. & 6.50 & 7.22 & 7.96 & 8 & 9.51 & 11.82 & 15.41 & 18.59 & 21.46 & 28.69 & 31.09 \\
\hline & & 4.67 & 4.81 & 5.01 & 5.36 & 5.5 & 5.7 & 6.11 & 6.87 & 7.64 & & 9.19 & 11.42 & 14.79 & 17.75 & 20.40 & 27.15 & 29.38 \\
\hline & & 4.33 & 4.45 & 4.64 & 4.98 & 5.1 & 5.33 & 5.70 & 6.45 & 7.2 & 7. & 8.69 & 10.79 & 13.91 & 16.63 & 19.06 & 25.23 & 27.32 \\
\hline & & 99 & 4.10 & 4.2 & 4.62 & 4.7 & 4.9 & 5.31 & 5.9 & 6.6 & & 8.02 & 9.91 & 12.70 & 15.14 & 17.31 & 22.94 & 24.92 \\
\hline & & 3.67 & 3.78 & 30 & 4.25 & 4.3 & 4.5 & 4.82 & 5.40 & 5.9 & 6 & 7.12 & 8.74 & 11.12 & 13.19 & 15.03 & 20.03 & 22.02 \\
\hline & & 3.35 & 3.44 & 3.58 & 3.81 & 3.91 & 4.05 & 4.28 & 4.75 & 5.20 & 5.63 & 6.07 & 7.28 & 9.11 & 10.72 & 12.19 & 16.67 & 18.78 \\
\hline & & 3.01 & 3.09 & 3.21 & 3.40 & 3.46 & 3.55 & 3.69 & 3.94 & 4.19 & 4. & 4.69 & 5.45 & 6.62 & 7.69 & 8.74 & 13.70 & $* * *$ \\
\hline 1.25 & 48 & 2.75 & 2.77 & 2.78 & 2.82 & 2.84 & 2.86 & 2.89 & 2.97 & 3.05 & 3.12 & 3.17 & 3.35 & 3.58 & 3.96 & 4.69 & 10.86 & *** \\
\hline
\end{tabular}

Notes. Surface rotation period is reported in days. In the isochrone at $4.57 \mathrm{Gyr}$, missing entries (indicated by $* * *$ ) correspond to stars that have already left the main sequence by that age.
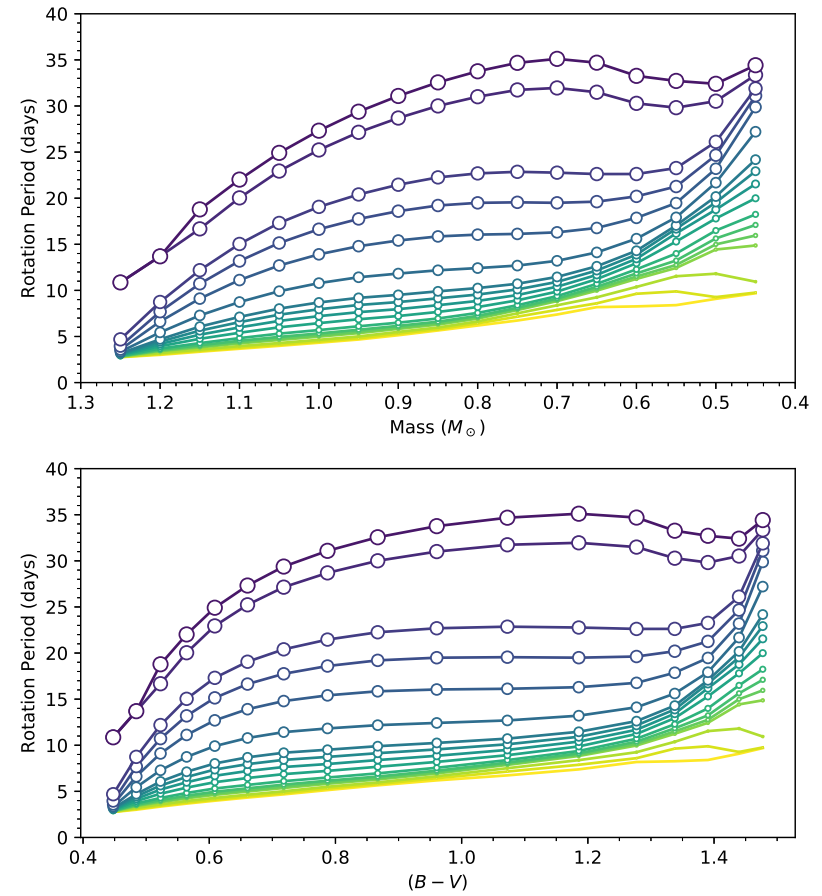

Fig. A.1. Rotational isochrones reported in Table A.1 (age range: $100 \mathrm{Myr}-4.57 \mathrm{Gyr})$. Top panel: rotation period vs. mass; bottom: rotation period vs. $(B-V)$ color. The size of the circles increases monotonically with age.

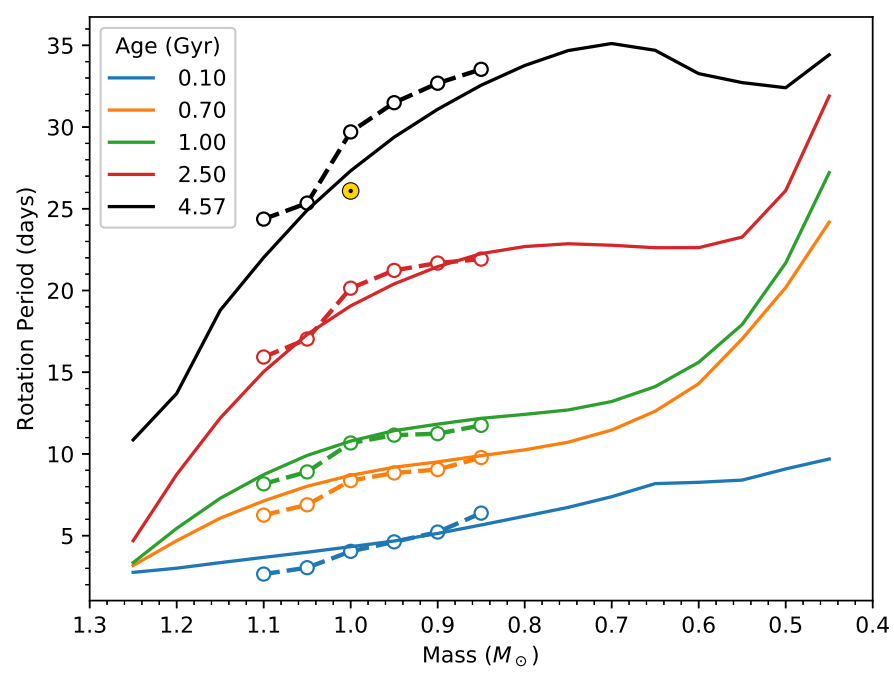

Fig. A.2. Rotational isochrones from this work (solid lines) compared with those of LS15 (dashed lines with circles). The Sun is also shown for reference. 